\title{
Uso de prácticas de Adaptación basada en Ecosistemas por pequeños cafetaleros en Centroamérica ${ }^{1}$
}

\section{Use of Ecosystem-based Adaptation practices by smallholder coffee farmers in Central America}

\author{
Adina Chain-Guadarrama², M.R. Martínez-Rodríguez, ${ }^{3,4}$, J.M. Cárdenas ${ }^{5}$, S. Vílchez-Mendoza ${ }^{6}$, C.A. Harvey
}

1 Recibido: 7 de marzo, 2018. Aceptado: 13 de junio, 2018. Este trabajo formó parte del proyecto de investigación CASCADA (Adaptación basada en Ecosistemas para pequeños productores de subsistencia y café en América Central), que forma parte de la Iniciativa Internacional por el Clima (ICI), financiada por el Ministerio Federal de Medio Ambiente, Protección de la Naturaleza, Obras Públicas y Seguridad Nuclear (BMUB) del Gobierno de la República de Alemania.

2 Consultora independiente. Turrialba 30501, Costa Rica. achain@ catie.ac.cr (https://orcid.org/0000-0002-6944-2064).

3 Conservation International. 2011 Crystal Drive, Suite 500, Arlington, VA, 22202, USA. celiaharvey@stanfordalumni.org (https://orcid. org/0000-0001-8015-703X).

4 ONU Medio Ambiente, Oficina Regional para América Latina y el Caribe, Panamá. ruth.martinez@un.org (https://orcid.org/0000-00024690-6794).

5 Ministerio de Agricultura y Ganadería (MAG). Agencia de extensión de Carara, Costa Rica. jcardenas@mag.go.cr

6 Centro Agronómico Tropical de Investigación y Enseñanza (CATIE). Turrialba 30501, Costa Rica. svilchez@catie.ac.cr (https://orcid. org/0000-0002-0326-551X).

\section{Resumen}

Introducción. En Centroamérica, los pequeños caficultores están amenazados por el aumento en las temperaturas y los cambios en los patrones de lluvia. Una estrategia para apoyar a las comunidades de pequeños agricultores a adaptarse al cambio climático es el uso de prácticas de Adaptación basada en Ecosistemas (AbE), las cuales integran la conservación, restauración y manejo sostenible de ecosistemas y biodiversidad. Sin embargo, existe poca información de cómo los productores utilizan estas prácticas. Objetivo. El objetivo de este estudio fue describir el uso de once prácticas de AbE entre pequeños agricultores de café, de cinco paisajes ubicados en Costa Rica, Honduras y Guatemala, así como comparar las características biofísicas y frecuencia de uso de las mismas entre paisajes. Materiales y métodos. La caracterización de las prácticas de AbE se realizó utilizando mapeo participativo, entrevistas, y mediciones de área y estructura y composición de la vegetación, entre julio 2014 y junio 2015. La comparación entre paisajes se realizó mediante un análisis de varianza. Resultados. Los resultados indican que muchos pequeños agricultores de café están implementando activamente el uso de prácticas AbE, entre ellas el uso de árboles de sombra en cafetales, los surcos en contorno, los huertos caseros y las cercas vivas. Sin embargo, existieron diferencias importantes entre paisajes en cuanto al uso y las características de AbE implementadas. Conclusión. Estas diferencias sugieren que los productores adaptan el uso de dichas prácticas en respuesta a los contextos sociales y biofísicos del medio en donde viven, y que existe valor en hacer intercambio de productores de diferentes regiones para compartir su conocimiento sobre estas prácticas.

Palabras clave: cerca viva, cambio climático, huertos familiares, sombra.

\begin{abstract}
Introduction. In Central America, smallholder coffee farmers are threatened by rising temperatures and changes in rainfall patterns. One strategy that could help support smallholder farmer communities adapt to climate change is the use of Ecosystem based Adaptation (EbA) practices, which are based on the conservation, restoration and


sustainable management of ecosystems and biodiversity. However, little information exists on how farmers use these practices. Objective. The objective of this work was to describe the use of eleven EbA practices by smallholder coffee farmers in five landscapes in Costa Rica, Honduras and Guatemala, and compare whether the biophysical characteristics and implementation of these practices differed between landscapes. Materials and methods. The characterization of EbA practices in the field was done using participatory mapping, surveys, area and measurements of vegetation composition and structure, from July 2014 to June 2015. Comparisons between landscapes were made using analysis of variance. Results. The study suggests that most farmers are already actively using EbA practices, especially shade trees in coffee plots, contour planting, home gardens and live fences. However, there were important differences across landscapes in the prevalence and characteristics of the EbA practices implemented. Conclusion. These differences suggest that smallholder farmers adapt the use of these practices in response to their social and biophysical contexts and that there is value in organizing exchanges among farmers from different regions to share their knowledge of these practices.

Keywords: climate change, home gardens, live fences, shade.

\section{Introducción}

A nivel mundial se estima que hay cerca de 500 millones de pequeños agricultores (Graeub et al., 2016), quienes dependen de pequeñas parcelas de agricultura para su seguridad alimentaria y sus medios de vida. Estos agricultores representan un grupo altamente vulnerable ante el cambio climático, principalmente porque dependen de la agricultura de secano, tienen terrenos ubicados en áreas remotas y marginales, y tienen un acceso restringido a asistencia técnica, crédito y apoyo institucional (Hellin y Schrader, 2003; Morton, 2007; Tucker et al., 2010; Vermeulen, 2014; Graeub et al., 2016), además, son altamente vulnerables a las amenazas climáticas (Baca et al., 2014; Hannah et al., 2017). Muchos cultivan café, que es muy sensible a cambios en la temperatura o en patrones de precipitación (Gay et al., 2006).

En la región centroamericana, existen aproximadamente 2,3 millones de pequeños agricultores (PRESANCA II y FAO, 2011), que se verán afectados por el cambio climático. Según los modelos climáticos, se espera que la magnitud del cambio climático sea mayor en la región centroamericana que en cualquier otra región tropical (Giorgi, 2006). En Centroamérica, se prevén incrementos en la temperatura, cambios en la distribución de lluvia y aumento en la frecuencia de eventos de lluvia de mayor intensidad (Aguilar et al., 2005; Neelin et al., 2006, Hannah et al. 2017). Como consecuencia, se espera que ocurran variaciones significativas en la disponibilidad de agua (Imbach et al., 2012; 2015), que haya un aumento en los brotes de ciertas plagas y patógenos (Avelino et al., 2015), y una reducción en la productividad de cultivos claves para los pequeños agricultores, incluyendo el café, el maíz y el frijol (Jones y Thornton, 2003; Baca et al., 2014).

En el caso particular del café, en Mesoamérica se espera una variación en la distribución de las tierras óptimas para su cultivo, como resultado de los cambios en el clima. Los modelos climáticos proyectan una disminución en la aptitud climática en altitudes más bajas y latitudes más altas, con la probabilidad de disminuir hasta en un 30\% el área apta total para el cultivo del café para el año 2050 (Jha et al., 2011; Baca et al., 2014; Bunn et al., 2015; Ovalle-Rivera et al., 2015). Los cambios en la cantidad y la sincronización de los eventos de lluvia afectarían también el crecimiento, la floración y la fructificación del café, y, por lo tanto, la producción total y la calidad del mismo (Villers et al., 2009; Peters y Carroll, 2012). Además, con respecto a las plagas y patógenos que afectan este cultivo, se sugiere que tanto la roya (Hemileia vastatrix) como la broca del café (Hypothenemus hampei) se beneficiarán de las temperaturas elevadas (Avelino et al., 2015; Magrach y Ghazoul, 2015) y del incremento en eventos de precipitación (Damon, 2000). En general, el cambio climático tendrá repercusiones negativas sobre los beneficios económicos derivados del café (Schroth et al., 2009) y sobre las comunidades rurales que dependen de este cultivo (Harvey et al., 2018). 
Actualmente, existen estrategias y acciones a nivel nacional e internacional para promover la adaptación agropecuaria ante el cambio climático (Dinesh et al., 2016). Entre estas opciones se encuentra el enfoque de Adaptación basada en Ecosistemas (AbE), definido como el uso de servicios ecosistémicos y biodiversidad como parte de una estrategia para ayudar a las personas a adaptarse a los impactos negativos del cambio climático. La AbE aplicada a la agricultura es una opción viable para promover la adaptación de los pequeños productores ante el cambio climático, especialmente porque no requiere mucha inversión o recursos para implementarse, en comparación a otras opciones de adaptación como la adopción de nuevas tecnologías que requieren insumos externos (ej. variedades de semillas mejoradas, sistemas de irrigación o mayor uso de agroquímicos) y que están fuera del alcance de muchos pequeños productores (Vignola et al., 2015).

A escala de parcela o finca, prácticas de AbE pueden incluir el uso de sistemas agroforestales para amortiguar los impactos de altas temperaturas, fuertes lluvias y otros efectos climáticos sobre cultivos o ganado (Lin, 2007; Verchot et al., 2007; Siles et al., 2010), y el establecimiento de cortinas rompevientos para reducir el impacto de vientos extremos (Rosenberg, 1992; Easterling et al., 1997). El uso de prácticas de conservación de suelo (ej. cobertura viva o terrazas) previene la erosión y mantiene la fertilidad bajo intensas lluvias (Dabney, 1998; Erenstein, 2003). El establecimiento de cercas vivas ayuda a prevenir la erosión del suelo y proveer forraje al ganado durante la época seca (Harvey et al., 2005), mientras que, la diversificación de cultivos, cultivares o razas de animales, ayuda a minimizar el riesgo de pérdida de producción debido a condiciones de clima cambiantes o brotes de plagas o patógenos asociados al clima (Lin, 2011; Burnham y Ma, 2015). A nivel de paisaje, algunos ejemplos de AbE son la conservación o restauración de bosques ribereños para mantener el flujo de cuerpos de agua bajo las condiciones cambiantes de lluvia (Capon et al., 2013), y la conservación de bosques en áreas montañosas para prevenir la erosión y los deslizamientos debidos a eventos de clima extremo (Locatelli et al., 2011).

A pesar del creciente interés en la importancia de la AbE para apoyar la adaptación climática de los pequeños productores, aún se necesita información sobre el uso, características y variación de estas prácticas en diferentes paisajes. El objetivo de este estudio fue describir el uso de once prácticas de AbE entre pequeños agricultores de café de cinco paisajes ubicados en Costa Rica, Honduras y Guatemala, así como comparar las características biofísicas y frecuencia de uso de las mismas entre paisajes. Esta información sobre el uso de diferentes prácticas de $\mathrm{AbE}$ por pequeños productores es relevante, ya que puede ayudar a guiar las políticas y estrategias de adaptación en la región (Donatti et al., 2017), al brindar información sobre la prevalencia de estas prácticas en diferentes paisajes y las oportunidades de aumentar su uso.

\section{Materiales y métodos}

\section{Selección de paisajes y finqueros}

Se caracterizaron once prácticas de AbE (detalladas más adelante) utilizadas por 191 pequeños productores de café en cinco paisajes centroamericanos: Turrialba y Los Santos en Costa Rica, Acatenango y Chiquimula en Guatemala, y Yoro en Honduras (Cuadro 1). Los paisajes de estudio fueron seleccionados con base en tres características principales: i) estar dominados por fincas de pequeños productores; ii) estar dominados por la producción de café, y iii) representar comunidades agrícolas con baja capacidad adaptativa al cambio climático. Estas comunidades tienen bajo acceso a mercados y créditos, poca mano de obra, problemas de tenencia de la tierra y mayores índices de migración. Detalles sobre el análisis de capacidad adaptativa en estos paisajes y su similitud con otros paisajes de pequeños productores en la región centroamericana pueden ser revisados en Holland et al. (2017). Adicionalmente, se eligieron paisajes localizados en zonas de vida de Holdridge contrastantes que abarcan desde bosque húmedo y muy húmedo subtropical hasta bosque pluvial premontano (Cuadro 1). De los cinco 
Cuadro 1. Características de los cinco paisajes estudiados en donde se caracterizó el uso de prácticas basadas en ecosistemas en pequeñas fincas cafetaleras. Costa Rica, Guatemala y Honduras, julio 2014 a junio 2015.

Table 1. Characteristics of the five landscapes where EbA practices were characterized in smallholder coffee farms. Costa Rica, Guatemala and Honduras, July 2014 to June 2015.

\begin{tabular}{|c|c|c|c|c|c|c|}
\hline & Características & $\begin{array}{l}\text { Turrialba, } \\
\text { Costa Rica }\end{array}$ & $\begin{array}{l}\text { Los Santos, } \\
\text { Costa Rica }\end{array}$ & $\begin{array}{c}\text { Acatenango, } \\
\text { Guatemala }\end{array}$ & $\begin{array}{c}\text { Chiquimula, } \\
\text { Guatemala }\end{array}$ & $\begin{array}{c}\text { Yoro, } \\
\text { Honduras }\end{array}$ \\
\hline \multirow{4}{*}{ 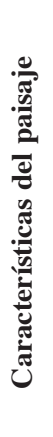 } & $\begin{array}{c}\text { Municipalidades } \\
\text { incluidas en la } \\
\text { encuesta }\end{array}$ & $\begin{array}{c}\text { Turrialba, Costa } \\
\text { Rica }\end{array}$ & $\begin{array}{l}\text { Dota, Tarrazú, } \\
\text { León Cortés }\end{array}$ & $\begin{array}{c}\text { Acatenango, } \\
\text { Alotenango, San } \\
\text { Pedro Yepocapa }\end{array}$ & $\begin{array}{c}\text { Quetzaltepeque, } \\
\text { San Jacinto, San } \\
\text { Juan Ermita }\end{array}$ & $\begin{array}{l}\text { Yoro, } \\
\text { Yorito, } \\
\text { Victoria }\end{array}$ \\
\hline & Área total (ha) & 158800 & 82000 & 42600 & 39600 & 325900 \\
\hline & $\begin{array}{l}\text { Zona de vida de } \\
\text { Holdridge }\end{array}$ & $\begin{array}{l}\text { Bosque muy } \\
\text { húmedo tropical } \\
\text { premontano; } \\
\text { Bosque pluvial } \\
\text { premontano }\end{array}$ & $\begin{array}{l}\text { Bosque muy } \\
\text { húmedo } \\
\text { montano bajo; } \\
\text { Bosque pluvial } \\
\text { premontano }\end{array}$ & $\begin{array}{l}\text { Bosque muy } \\
\text { húmedo } \\
\text { subotropical }\end{array}$ & $\begin{array}{l}\text { Bosque húmedo } \\
\text { subtropical }\end{array}$ & $\begin{array}{l}\text { Bosque muy } \\
\text { húmedo } \\
\text { subtropical; } \\
\text { Bosque húmedo } \\
\text { subtropical }\end{array}$ \\
\hline & $\begin{array}{c}\text { Principal actividad } \\
\text { agrícola }\end{array}$ & Café & Café & $\begin{array}{l}\text { Café y granos } \\
\text { básicos }\end{array}$ & $\begin{array}{l}\text { Café y granos } \\
\text { básicos }\end{array}$ & $\begin{array}{l}\text { Café y granos } \\
\text { básicos }\end{array}$ \\
\hline
\end{tabular}

paisajes seleccionados, los paisajes de Turrialba y Los Santos están dominados por producción de café, mientras que Yoro, Chiquimula y Acatenango incluyen una mezcla de producción de café y granos básicos. Todos los cinco paisajes son paisajes cafetaleros típicos a lo largo de la región centroamericana, con pequeñas fincas y finqueros con bajos niveles educativos y baja capacidad adaptativa (Holland et al., 2017).

La selección de productores en cada paisaje se realizó con base en información previa obtenida a través de una encuesta sobre la finca, hogar y uso de prácticas de AbE, realizada a 860 hogares de pequeños productores de café y granos básicos seleccionados al azar en cada paisaje. Más detalles sobre el marco de muestreo de esta encuesta pueden ser revisado en Harvey et al. (2017; 2018). Específicamente, los productores fueron seleccionados en cada paisaje con base en información recolectada sobre el número de prácticas AbE utilizadas por finca, estratificándolos en dos grupos de alta y baja frecuencia de uso, y seleccionando al azar veinticinco productores de cada grupo, de manera que en cada paisaje hubiera una representación adecuada de la diversidad de fincas (Harvey et al., 2017). De este conjunto de productores, para este estudio se seleccionaron solo aquellos que producen café, para un total de 191 productores distribuidos en los cinco paisajes seleccionados: Turrialba ( $\mathrm{n}=50$ fincas), Los Santos $(\mathrm{n}=50$ ), Acatenango $(n=42)$, Chiquimula $(n=20)$ y Yoro $(n=29)$.

\section{Caracterización de las prácticas de AbE}

Se caracterizó el uso de once prácticas AbE listadas a continuación: uso de sombra en parcelas de café, cercas vivas, cortinas rompevientos, huertos caseros, terrazas, surcos en contorno, cobertura viva, barbechos, bosques ribereños, parches de bosque y plantaciones forestales (Figura 1). Esta lista de prácticas de AbE se basó en una revisión extensiva de literatura sobre prácticas comúnmente utilizadas por pequeños productores de café y granos básicos (Bautista-Solís et al., 2014). Para caracterizar el uso de estas prácticas en cada una de las fincas seleccionadas, primero se empleó un método de mapeo participativo con los productores, que permitió localizar las parcelas individuales en la finca y caracterizar el uso de suelo (ej. campos de cultivo, potreros, barbechos, áreas forestales, cuerpos de agua, etc.). Para poder visitar dichas áreas y caracterizar las prácticas a través de trabajo de campo, se solicitó a los productores que identificaran cuáles prácticas de $\mathrm{AbE}$ tenían por parcela individual y que indicaran en el mapa su sitio de implementación, registrando así la presencia/ausencia de cada una de las once prácticas. 


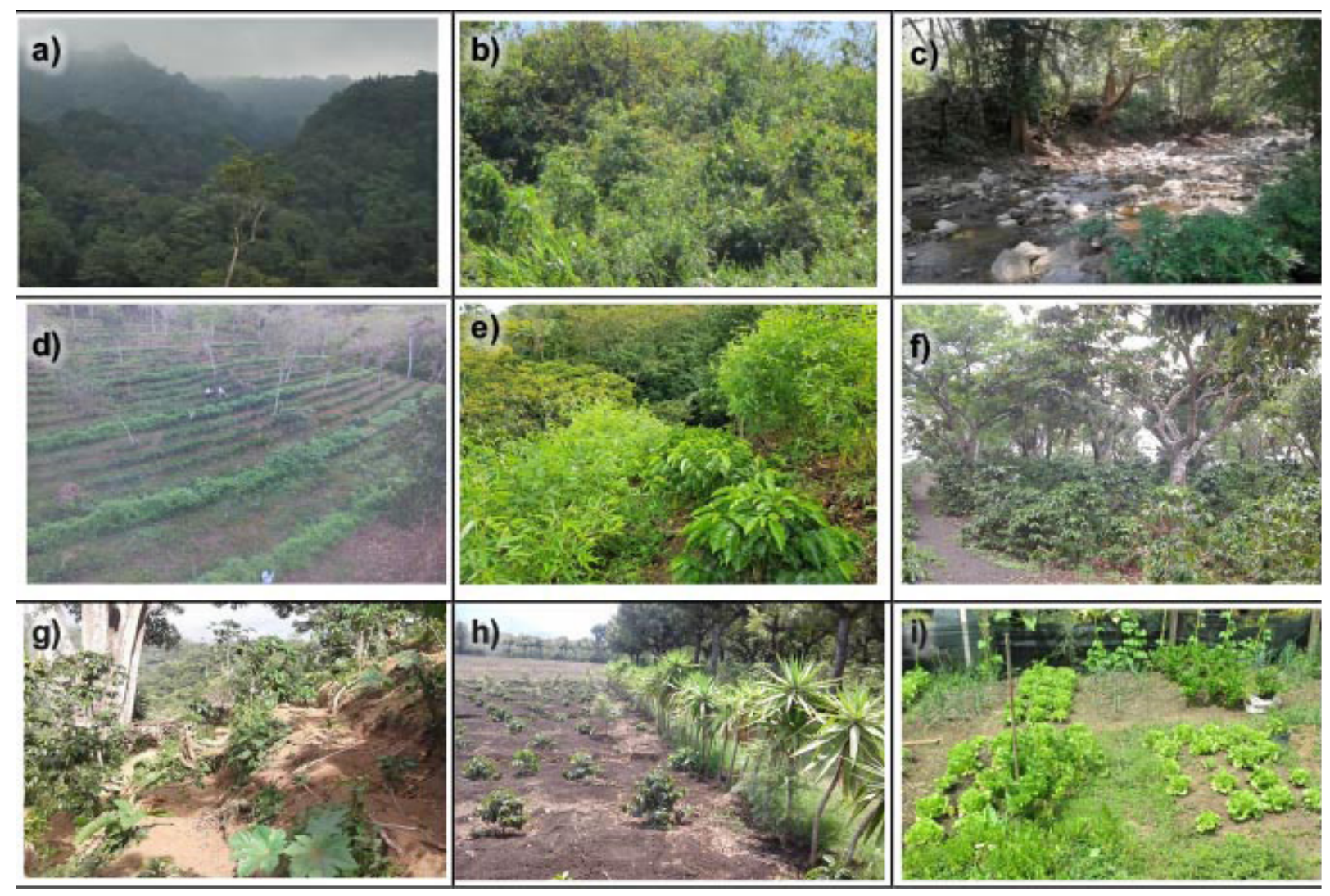

Figura 1. Diferentes prácticas de AbE utilizadas por pequeños productores de café en cinco paisajes centroamericanos. Turrialba y Los Santos en Costa Rica, Acatenango y Chiquimula en Guatemala, y Yoro en Honduras. Julio 2014 a junio 2015.

a) bosque; b) barbecho; c) bosque de río; d) cultivo a contorno; e) cobertura viva; f) sombra, g) terrazas; h) cercas vivas; i) huerto casero.

Figure 1. Different EbA practices used by smallholder coffee farmers in five Central American landscapes. Turrialba and Los Santos in Costa Rica, Acatenango and Chiquimula in Guatemala, and Yoro in Honduras. July 2014 to June 2015.

a) forest; b) fallow; c) riparian forest; d) contour planting; e) cover crops; f) shaded coffee; g) terraces; h) live fences; i) home gardens.

Para cada una de las prácticas AbE identificadas en las fincas se obtuvo información detallada sobre su implementación a través de mediciones directas en campo. Para bosques, bosques ribereños, huertos caseros, cortinas rompevientos y barbechos, se midió el área de todas las parcelas y se calculó el área total bajo esta práctica a nivel de la finca. Para la caracterización del uso de prácticas de AbE dentro de parcelas de café, se seleccionaron al azar hasta tres parcelas por finca. En el centro de cada parcela se estableció un área de 20 x $50 \mathrm{~m}\left(100 \mathrm{~m}^{2}\right)$, donde se identifico a nivel de especies y se midió el diámetro a la altura del pecho (dap) de todos los árboles de sombra. Para las cercas vivas, se seleccionaron aleatoriamente hasta seis cercas vivas por finca y se registraron todos los árboles $>5 \mathrm{~cm}$ dentro de un segmento de $100 \mathrm{~m}$ de la cerca, elegido al azar, se identificaron sus especies y se midió su dap. Para estas dos prácticas la información obtenida fue resumida en términos del promedio de especies por parcela, el dap promedio por parcela, y la densidad arbórea promedio (árboles/ha). También se registró si el productor utiliza o no cobertura viva, terrazas y surcos en contorno en las parcelas. Todos los datos fueron colectados en campo 
usando tabletas programadas con el software SurveyCTO, un producto que captura, registra y procesa los datos colectados durante entrevistas estructuradas y trabajo de campo. El trabajo de campo se realizó de julio de 2014 hasta junio de 2015 .

\section{Análisis de datos}

Se empleó estadística descriptiva para caracterizar la prevalencia de las prácticas de AbE a través de las fincas y paisajes, para obtener el porcentaje de fincas con prácticas de AbE individuales y el número total de ellas por finca. Además, se comparó el área promedio destinada para las prácticas de bosques, bosques ribereños, huertos caseros, cortinas rompevientos y barbechos, usando análisis de la varianza (ANDEVA). Los promedios de densidad arbórea, número de especies y diámetro arbóreo en las parcelas de café y cercas vivas, también se compararon entre paisajes con ANDEVAS. Los paisajes con una frecuencia menor a cinco productores por práctica, no fueron tomados en cuenta en estos análisis comparativos. También se describió por paisaje la composición de especies de los árboles de sombra en los cafetales y en las cercas vivas. Todos los análisis se realizaron en el paquete estadístico InfoStat (Di-Rienzo et al., 2016).

\section{Resultados}

\section{Características de los productores y fincas de café}

Los datos colectados a través de las entrevistas indicaron que del total de productores de café ( $n=191)$ el 82,7\% fueron hombres (Cuadro 2). En Acatenango, Chiquimula y Yoro el tamaño promedio de las familias productoras fue significativamente mayor que en los dos paisajes de Costa Rica $\left(\mathrm{F}_{4,186}=13,8 ; \mathrm{p}<0,0001\right)$. El 46,1\% de los productores entrevistados tuvo un nivel de educación menor al grado de primaria, $38,2 \%$ un nivel de educación primaria completa, y solo el 15,7\% un nivel de educación más elevado (Cuadro 2). Los productores de los paisajes de Turrialba y Los Santos presentaron los mayores niveles de educación en comparación con el resto de los paisajes estudiados. Además, los pequeños productores entrevistados en Turrialba tuvieron mayor número de años de experiencia productiva, que los productores de Los Santos, Acatenango y Yoro $\left(\mathrm{F}_{4,186}=4,8, \mathrm{p}=0,001\right)$. Finalmente, $60 \%$ de los agricultores de Los Santos reportaron haber recibido capacitación técnica en los últimos dos años, casi el doble de los productores que así lo indicaron en Yoro y casi tres veces más que el porcentaje de productores en Acatenango. Además, el 70\% de productores en Los Santos reportaron haber recibido consejo por parte de un agrónomo en los últimos dos años, muy por arriba de los productores en el resto de los paisajes estudiados (Cuadro 2).

El tamaño promedio de las fincas de café entre los cinco paisajes fue de 2,6 ha $( \pm 0,03)$. Las fincas de los productores de Los Santos fueron más grandes en comparación con el resto de los paisajes $\left(\mathrm{F}_{4,186}=7,1, \mathrm{p}<0,0001\right.$; Cuadro 2). Mientras que los productores de Turrialba y Los Santos solo cultivaron café, el $85 \%$ y el $93 \%$ de los productores en Chiquimula y Yoro, respectivamente, también reportaron cultivar granos básicos (ya sea maíz, frijol o ambos). En contraste, solo el 17\% de productores de café en Acatenango sembraron granos básicos. El tamaño promedio de las parcelas de café fue de 0,8 ha $( \pm 0,02)$ entre todos los productores entrevistados. Las parcelas de café más grandes se ubicaron en el paisaje de Los Santos $\left(\mathrm{F}_{4,186}=57,40, \mathrm{p}<0,0001\right)$ en comparación con el resto de los paisajes (Cuadro 2). Los productores de Guatemala presentaron los menores tamaños de área dedicada al cultivo de café (Cuadro 2). En cuanto a la tenencia de la tierra, la mayoría de los productores $(97,9 \%)$ entrevistados son dueños de al menos una porción de la tierra que cultivan. 
Cuadro 2. Características de los pequeños productores de café centroamericanos y las fincas en donde se caracterizó el uso de prácticas de Adaptación basada en Ecosistemas. Costa Rica, Guatemala y Honduras. Julio 2014 a junio 2015.

Table 2. Characteristics of the Central America smallholder coffee farmers and farms where Ecosystem-based Adaptation practices were characterized. Costa Rica, Guatemala and Honduras. July 2014 to June 2015.

\begin{tabular}{|c|c|c|c|c|c|c|c|}
\hline & Características & $\begin{array}{l}\text { Turrialba, } \\
\text { Costa Rica }\end{array}$ & $\begin{array}{l}\text { Los Santos, } \\
\text { Costa Rica }\end{array}$ & $\begin{array}{c}\text { Acatenango, } \\
\text { Guatemala }\end{array}$ & $\begin{array}{c}\text { Chiquimula, } \\
\text { Guatemala }\end{array}$ & $\begin{array}{c}\text { Yoro, } \\
\text { Honduras }\end{array}$ & Total \\
\hline \multicolumn{2}{|c|}{ n (número de productores encuestados) } & 50 & 50 & 42 & 20 & 29 & 191 \\
\hline \multirow{4}{*}{ 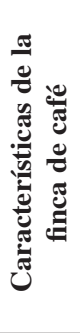 } & $\begin{array}{l}\text { Tamaño (ha) promedio de todas las } \\
\text { fincas }( \pm \text { E.E) }\end{array}$ & $2,2 \pm 0,10 \mathrm{~b}$ & $6,0 \pm 0,19$ a & $1,50,12 \mathrm{~b}$ & $1,4 \pm 0,14 \mathrm{~b}$ & $2,0 \pm 0,15 \mathrm{~b}$ & $2,6 \pm 0,03$ \\
\hline & $\begin{array}{l}\text { Tamaño promedio (ha) de las } \\
\text { parcelas de café }\end{array}$ & $0,9 \pm 0,07 \mathrm{~b}$ & $1,8 \pm 0,10 \mathrm{a}$ & $0,1 \pm 0,01 \mathrm{~d}$ & $0,2 \pm 0,03 \mathrm{c}$ & $0,8 \pm 0,06 \mathrm{~b}$ & $0,8 \pm 0,02$ \\
\hline & $\begin{array}{l}\text { \# y }(\%) \text { de productores de café que } \\
\text { además siembran granos básicos }\end{array}$ & 0 & 0 & $7(17)$ & $17(85)$ & $27(93)$ & $51(26,7)$ \\
\hline & $\begin{array}{l}\text { Distancia promedio en } \mathrm{km} \text { de la casa } \\
\text { la finca ( } \pm \text { E.E.) }\end{array}$ & $0,37 \pm 0,06 \mathrm{c}$ & $1,77 \pm 0,10 \mathrm{~b}$ & $3,96 \pm 0,11 \mathrm{a}$ & $1,59 \pm 0,13 b$ & $1,08 \pm 0,1 \mathrm{~b}$ & $1,48 \pm, 03$ \\
\hline \multirow{8}{*}{ 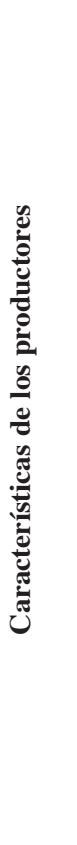 } & $\begin{array}{l}\text { Tamaño promedio de la familia ( } \pm \\
\text { E.E.) }\end{array}$ & $3,3 \pm 0,27 b$ & $3,60 \pm 0,27 \mathrm{~b}$ & $5,6 \pm 0,29$ a & $5,0 \pm 0,43 \mathrm{a}$ & $5,6 \pm 0,35$ a & $4,4 \pm 0,07$ \\
\hline & $\begin{array}{l}\text { Son dueños de al menos un pedazo } \\
\text { de tierra que cultivan }\end{array}$ & $50(100)$ & $50(100)$ & $39(92,9)$ & $19(95)$ & $29(100)$ & $187(97,9)$ \\
\hline & $\begin{array}{l}\text { Número promedio de años de } \\
\text { experiencia como productor }( \pm \text { E.E) }\end{array}$ & $41,2 \pm 2,36$ a & $30,4 \pm 2,36 b$ & $29,5 \pm 2,57 \mathrm{~b}$ & $34,6 \pm 3,73 \mathrm{ab}$ & $\begin{array}{c}27,0 \pm 3,10 \\
\mathrm{~b}\end{array}$ & $33,0 \pm, 38$ \\
\hline & $\begin{array}{l}\text { \# y }(\%) \text { de productores que } \\
\text { recibieron capacitación en los } \\
\text { últimos dos años }\end{array}$ & $3(6)$ & $30(60)$ & $9(21,4)$ & $5(25)$ & $11(37,9)$ & $58(30,4)$ \\
\hline & $\begin{array}{l}\text { \# y }(\%) \text { de productores que } \\
\text { recibieron consejo de un agrónomo } \\
\text { en los últimos dos años }\end{array}$ & $14(28)$ & $35(70)$ & $7(16,7)$ & $1(5)$ & $2(6,9)$ & $59(30,9)$ \\
\hline & $\begin{array}{l}\text { \# y }(\%) \text { de productores que no } \\
\text { tienen un nivel de educación } \\
\text { primaria completo }\end{array}$ & $19(38)$ & $5(10)$ & $27(64,3)$ & $13(65)$ & $24(82,8)$ & $88(46,1)$ \\
\hline & $\begin{array}{l}\text { \# y }(\%) \text { de productores que solo } \\
\text { tienen nivel de educación primaria } \\
\text { completado }\end{array}$ & $25(50)$ & $32(64)$ & $7(16,7)$ & $5(25)$ & $4(13,8)$ & $73(38,2)$ \\
\hline & $\begin{array}{l}\text { \# y }(\%) \text { de productores que tienen } \\
\text { más que solo el nivel de educación } \\
\text { primaria completado }\end{array}$ & $6(12)$ & $13(26)$ & 8 (19) & $2(10)$ & $1(3,4)$ & $30(15,7)$ \\
\hline
\end{tabular}

Se reportan medias ajustadas y errores estándar (E.E). Letras asociadas a las medias indican diferencias estadísticas entre paisajes, basado en ANDEVA $(\mathrm{p}<0,05)$ / Adjusted means and standard errors (E.E) are reported. Letters associated with means indicate statistical differences across landscapes, based on ANOVA $(\mathrm{p}<0.05)$.

\section{Uso de las prácticas de AbE en las fincas}

A través de trabajo de campo se caracterizaron 191 fincas de café y se midió un total de 292 parcelas con ese cultivo (incluyendo mediciones de 7665 árboles de sombra), 409 segmentos de cercas vivas (incluyendo mediciones de 5076 árboles), 47 fragmentos de bosque (432,75 ha), 18 bosques ribereños (6,67 ha), 59 barbechos (34,32 ha), y tres plantaciones forestales (0,6 ha). 
Los pequeños productores de café usaron en promedio 3,68 $\pm 0,10$ prácticas de AbE por finca, con un rango de 1 a 7 por finca (Cuadro 3). Los paisajes con mayor número promedio de prácticas de AbE usadas por los productores fueron Los Santos y Yoro, mientras que, en los paisajes de Guatemala fue donde se observaron menos (Cuadro 3; $\left.\mathrm{F}_{4,186}=40,69, \mathrm{p}<0,0001\right)$. La práctica más usada por los productores fue árboles de sombra $(94,2 \%$ del total de productores), seguida por surcos en contorno $(74,9 \%)$, huertos caseros $(56 \%)$ y cercas vivas $(49,2 \%)$ (Cuadro 3). Las prácticas menos comunes fueron el uso de cobertura viva, cortinas rompevientos y la presencia de plantaciones forestales, usadas por menos del 3\% de los entrevistados (Cuadro 3).

Cuadro 3. Número y porcentaje $(\%)$ de pequeños productores de café que usan diferentes prácticas de Adaptación basada en Ecosistemas en cinco paisajes de Centroamérica. Los datos se presentan en orden decreciente de abundancia. Costa Rica, Guatemala y Honduras. Julio 2014 a junio 2015.

Table 3. Number and percentage (\%) of smallholder coffee farmers using different Ecosystem-based Adaptation practices in five Central American landscapes. Data is presented in decreasing order of abundance. Costa Rica, Guatemala and Honduras. July 2014 to June 2015.

\begin{tabular}{|c|c|c|c|c|c|c|}
\hline & $\begin{array}{l}\text { Turrialba, } \\
\text { Costa Rica }\end{array}$ & $\begin{array}{l}\text { Los Santos, } \\
\text { Costa Rica }\end{array}$ & $\begin{array}{c}\text { Acatenango, } \\
\text { Guatemala }\end{array}$ & $\begin{array}{c}\text { Chiquimula, } \\
\text { Guatemala }\end{array}$ & $\begin{array}{c}\text { Yoro, } \\
\text { Honduras }\end{array}$ & $\begin{array}{c}\text { Total } \\
\text { (6 paisajes) }\end{array}$ \\
\hline Prácticas de AbE & $\mathrm{n}=50$ & $\mathrm{n}=50$ & $\mathrm{n}=42$ & $\mathrm{n}=20$ & $\mathrm{n}=29$ & $\mathrm{n}=191$ \\
\hline $\begin{array}{l}\text { Árboles de sombra en } \\
\text { parcelas de café }\end{array}$ & $47(94)$ & $48(96)$ & $40(95,2)$ & $20(100)$ & $25(86,2)$ & $180(94,2)$ \\
\hline Surcos en contorno & $23(46)$ & $49(98)$ & $40(95,2)$ & $4(20)$ & $27(93,1)$ & $143(74,9)$ \\
\hline Huerto casero & $34(68)$ & $33(66)$ & $3(7,1)$ & $8(40)$ & $29(100)$ & $107(56,0)$ \\
\hline Cercas vivas & $38(76)$ & $20(40)$ & $2(4,8)$ & $15(75)$ & $19(65,5)$ & $94(49,2)$ \\
\hline Terrazas & $10(20)$ & $42(84)$ & $4(9,5)$ & $3(15)$ & 0 & $59(30,9)$ \\
\hline Barbecho & $7(14)$ & $15(30)$ & $3(7,1)$ & $1(5)$ & $22(75,9)$ & $48(25,1)$ \\
\hline Bosque & $10(2)$ & $18(36)$ & 0 & $1(5)$ & $14(48,3)$ & $43(22,5)$ \\
\hline Bosque ribereño & $9(18)$ & $4(8)$ & 0 & 0 & $5(17,2)$ & $18(9,4)$ \\
\hline Cobertura viva & $1(2)$ & 0 & 0 & 0 & $1(3,5)$ & $5(2,6)$ \\
\hline Plantación forestal & $2(4)$ & 0 & 0 & $1(5,0)$ & 0 & $3(1,6)$ \\
\hline Cortina rompeviento & 0 & $1(2)$ & $2(4,8)$ & 0 & 0 & $3(1,6)$ \\
\hline $\begin{array}{l}\text { Número promedio de } \\
\text { prácticas }( \pm \mathrm{EE}) \text { por finca }\end{array}$ & $3,62 \pm 0,15 \mathrm{~b}$ & $4,60 \pm 0,15 \mathrm{a}$ & $2,31 \pm 0,16 \mathrm{c}$ & $2,65 \pm 0,24 \mathrm{c}$ & $4,90 \pm 0,20 \mathrm{a}$ & $3,68 \pm 0,07$ \\
\hline
\end{tabular}

Se reportan medias ajustadas y errores estándar (E.E). Letras asociadas a las medias indican diferencias estadísticas entre paisajes, basado en ANDEVA $(\mathrm{p}<0,05)$ / Adjusted means and standard errors (EE) are reported. Letters associated with means indicate statistical differences across landscapes, based on ANOVA $(\mathrm{p}<0.05)$.

El uso de árboles de sombra fue una práctica común a través de todos los paisajes, pero se observaron diferencias en la frecuencia de uso de otras prácticas entre paisajes (Cuadro 3). Así en Los Santos, Acatenango y Yoro, más del 93\% de los productores utilizaron surcos en contorno, pero solo el $46 \%$ y el $20 \%$ de los productores en Turrialba y Chiquimula hicieron uso de esta práctica. Además, mientras que el 100\% de los productores en Yoro reportó el uso de huertos caseros, solo el 7,1\% de los productores en Acatenango reportaron tener huertos caseros en sus fincas. Acatenango también se distinguió de los demás paisajes por la baja frecuencia en el uso de cercas vivas $(4,8 \%)$, terrazas $(9,5 \%)$ y barbechos $(7,1 \%)$. Los productores de Chiquimula también reportaron con poca frecuencia la presencia de barbechos (5\%), y los productores de Yoro no reportaron el uso de terrazas. En cambio, 
más del $80 \%$ de los productores en Los Santos usaron terrazas. Finalmente, la presencia de áreas de bosque en las fincas fue mayormente reportada entre los productores de Yoro $(48,3 \%)$ y Los Santos $(36 \%)$, mientras que, en Acatenango ningún productor de café reportó tener áreas de bosque en su finca (Cuadro 3).

\section{Características de las prácticas de AbE en las fincas}

\section{a) Árboles de sombra}

El trabajo en campo permitió caracterizar los árboles de sombra en las parcelas de café y los árboles en las cercas vivas. Del total de 292 parcelas de café, en 278 de ellas se registró el uso de árboles de sombra con una densidad promedio de 219,2 $( \pm 1,02)$ árboles/hectárea (Cuadro 4). La mayor densidad arbórea se observó en las fincas de café del paisaje de Los Santos, con más de 280 árboles/ha en promedio, y la menor densidad en los paisajes de Yoro (173 árboles/ha) y Turrialba (176 árboles/ha) $\left(\mathrm{F}_{4,175}=3,8 ; \mathrm{p}=0,0055\right)$. Los cafetales de Chiquimula y Yoro tuvieron los mayores valores de riqueza de especies de árboles se sombra $\left(\mathrm{F}_{4,175}=28,23 ; \mathrm{p}<0,0001\right)$, aún cuando para Yoro se observaron densidades de árboles más bajas en comparación con el resto de los paisajes (Cuadro 4). Las fincas de Los Santos y Acatenango tuvieron valores de riqueza de especies arbóreas similares entre ellos y menores a Chiquimula y Yoro, pero por encima de los cafetales estudiados en Turrialba. En promedio, los árboles de sombra en los cafetales, en los cinco paisajes estudiados, presentaron un diámetro a la altura del pecho

Cuadro 4. Características de los árboles de sombra en las parcelas de las fincas de pequeños productores de café en cinco paisajes de Centroamérica. Costa Rica, Guatemala y Honduras. Julio 2014 a junio 2015.

Table 4. Characteristics of shade trees in plots present in smallholder coffee farms in five Central American landscapes. Costa Rica, Guatemala and Honduras. July 2014 to June 2015.

\begin{tabular}{|c|c|c|c|c|c|c|}
\hline & $\begin{array}{l}\text { Turrialba, } \\
\text { Costa Rica }\end{array}$ & $\begin{array}{l}\text { Los Santos, } \\
\text { Costa Rica }\end{array}$ & $\begin{array}{c}\text { Acatenango, } \\
\text { Guatemala }\end{array}$ & $\begin{array}{c}\text { Chiquimula, } \\
\text { Guatemala }\end{array}$ & $\begin{array}{c}\text { Yoro, } \\
\text { Honduras }\end{array}$ & $\begin{array}{c}\text { Total }(5 \\
\text { paisajes })\end{array}$ \\
\hline n (parcelas) & 60 & 99 & 52 & 24 & 43 & 278 \\
\hline Densidad arbórea/ha ( \pm EE) & $176,0 \pm 1,1 \mathrm{~b}$ & $283,7 \pm 1,1 \mathrm{a}$ & $223,8 \pm 1,1 \mathrm{ab}$ & $259,5 \pm 1,2 \mathrm{ab}$ & $173,2 \pm 1,2 \mathrm{~b}$ & $219,2 \pm 1,0$ \\
\hline $\begin{array}{l}\text { Riqueza de especies/parcela } \\
( \pm \text { EE })\end{array}$ & $2,4 \pm 1,1 \mathrm{c}$ & $5,4 \pm 1,1 \mathrm{~b}$ & $5,7 \pm 1,1 \mathrm{~b}$ & $8,9 \pm 1,1 \mathrm{a}$ & $8,6 \pm 1,1 \mathrm{a}$ & $4,9 \pm 1,0$ \\
\hline $\begin{array}{l}\text { Diámetro de árboles (dap; } \\
\mathrm{cm})( \pm \mathrm{EE})\end{array}$ & $17,9 \pm 1,1 \mathrm{a}$ & $14,2 \pm 1,1 \mathrm{bc}$ & $18,5 \pm 1,1 \mathrm{a}$ & $11,4 \pm 1,1 \mathrm{c}$ & $16,3 \pm 1,1 \mathrm{ab}$ & $16,0 \pm 1,0$ \\
\hline n (número de individuos) & 1434 & 3273 & 1301 & 774 & 883 & 7665 \\
\hline $\begin{array}{l}\text { Diez especies más comunes } \\
\text { (\% del total de árboles) }\end{array}$ & $\begin{array}{c}\text { Erythrina } \\
\text { poeppigiana } \\
(78,5) ; \text { Cordia } \\
\text { alliodora } \\
(14,1) ; \\
\text { cítricos }(1,3) ; \\
\text { Solanum sp. } \\
(0,8) ; \text { Psidium } \\
\text { guajava }(0,7) ; \\
\text { Psidium } \\
\text { friedrichsthalia } \\
(0,5)\end{array}$ & $\begin{array}{c}\text { Erythrina } \\
\text { poeppigiana } \\
\text { (51,3); } \\
\text { Erythrina } \\
\text { fusca }(18,1) ; \\
\text { Persea } \\
\text { americana } \\
(8,43) ; \\
\text { Erythrina } \text { sp. } \\
(1,9) ; \text { Psidium } \\
\text { guajava }(1,6) ; \\
\text { cítricos }(1,5)\end{array}$ & $\begin{array}{c}\text { Grevillea } \\
\text { robusta }(37,2) ; \\
\text { Inga } \text { sp. } \\
(25,4) ; \text { Persea } \\
\text { americana } \\
(8,3) ; \text { Diphysa } \\
\text { americana } 4,0) ; \\
\text { Citharexylum } \\
\text { donnell-smithii } \\
(2,8) ; \text { Cedrela } \\
\text { sp. }(2,5)\end{array}$ & $\begin{array}{c}\text { Yucca } \\
\text { guatemalensis } \\
(9,4) ; \text { Cítricos } \\
(9,2) ; \text { Vernonia } \\
\text { leiocarpa }(7,2) ; \\
\text { Mangifera } \\
\text { indica }(6,6) ; \\
\text { Cedrela } \mathrm{sp}, \\
(6,2) ; \text { Diphysa } \\
\text { americana } \\
(4,9)\end{array}$ & $\begin{array}{c}\text { Inga sp. }(22,8) ; \\
\text { Mulule }(10,8) ; \\
\text { Cedrela sp. } \\
(4,8) ; \text { Vismia } \\
\text { baccifera } \\
(4,4) ; \text { Persea } \\
\text { americana } \\
(4,3) ; \\
\text { Perymenium } \\
\text { grande }(3,9)\end{array}$ & $\begin{array}{c}\text { Erythrina } \\
\text { poeppigiana } \\
(36,6) ; \\
\text { Erythrina fusca } \\
(7,8) ; \text { Inga } \mathrm{sp} . \\
(7,7) ; \text { Grevillea } \\
\text { robusta }(7,0) ; \\
\text { Persea } \\
\text { americana } \\
(5,8) ; \text { Cordia } \\
\text { alliodora }(3,3)\end{array}$ \\
\hline
\end{tabular}

Se reportan medias ajustadas y errores estándar (E.E). Letras asociadas a las medias indican diferencias estadísticas entre paisajes, basado en ANDEVA $(\mathrm{p}<0,05)$ / Adjusted means and standard errors $(\mathrm{EE})$ are reported. Letters associated with means indicate statistical differences across landscapes, based on ANOVA $(\mathrm{p}<0.05)$. 
(dap) de 16,0 cm $( \pm 1,01)$. En las fincas cafetaleras de Acatenango y Turrialba se pudieron observar árboles de tamaños más grandes en comparación a Los Santos y Chiquimula $\left(\mathrm{F}_{4,175}=6,33 ; \mathrm{p}=0,0001\right)$.

Entre los 7665 árboles de sombra medidos en todas las parcelas de café, se reconocieron 196 especies. La especie más común fue Erythrina poeppigiana (de nombre común poró), la cual correspondió al 36,6\% del total. Las especies E. fusca (bucaro o poro copey), Inga (caspirol, chalum, cuje, cushin o guama), Grevillea robusta (gravilea) y Persea americana (aguacate), fueron las especies más frecuentes después del poró, cada una de ellas con más de 400 árboles medidos en todas las parcelas de café (Cuadro 4). En las fincas de café de Turrialba y de Los Santos dominaron los árboles de E. poeppigiana. En Los Santos otras especies frecuentes fueron el bucaro y el aguacate, mientras que, en Turrialba fueron Cordia alliodora (laurel) y diferentes especies agrupadas como cítricos (Cuadro 4). En Acatenango, las tres especies más dominantes en orden descendiente fueron gravilea, caspirol y aguacate. En Chiquimula Yucca guatemalensis (izote o itabo), varias especies agrupadas como cítricos y Vernonia leiocarpa (suchini, suquinay o zukinay), fueron las más comunes. Finalmente, en Yoro las tres especies más dominantes fueron el caspirol, el mulule (de nombre científico desconocido) y la especie Cedrela sp. (cedro) (Cuadro 4).

La mayoría de los árboles de sombra medidos en las parcelas fueron reportados por los productores como árboles de servicio (80\%), especies que brindan algún servicio ecosistémico como sombra, materia orgánica, leña, refugio para la fauna, fijación de carbono y/o belleza escénica (Figura 2). Además, el 14,5\% correspondieron a árboles frutales y $6,2 \%$ a árboles maderables. Entre todos los árboles medidos, menos del $1 \%$ correspondieron a palmas u ornamentales. En Turrialba se observó la mayor proporción de árboles maderables $(15,2 \%)$, mientras que en las fincas de Los Santos fueron menos del 1\% (Figura 2). Por el contrario, en las fincas de Turrialba se encontró la menor proporción de árboles frutales $(3,4 \%)$ y en Chiquimula la mayor $(35,7 \%)$. La cantidad de árboles de servicio fue relativamente alta a través de todos los paisajes, pero se observó una mayor proporción en Los Santos $(84,5 \%)$ y Turrialba $(81,4 \%)$, en comparación con Chiquimula $(54,8 \%$, Figura 2$)$.
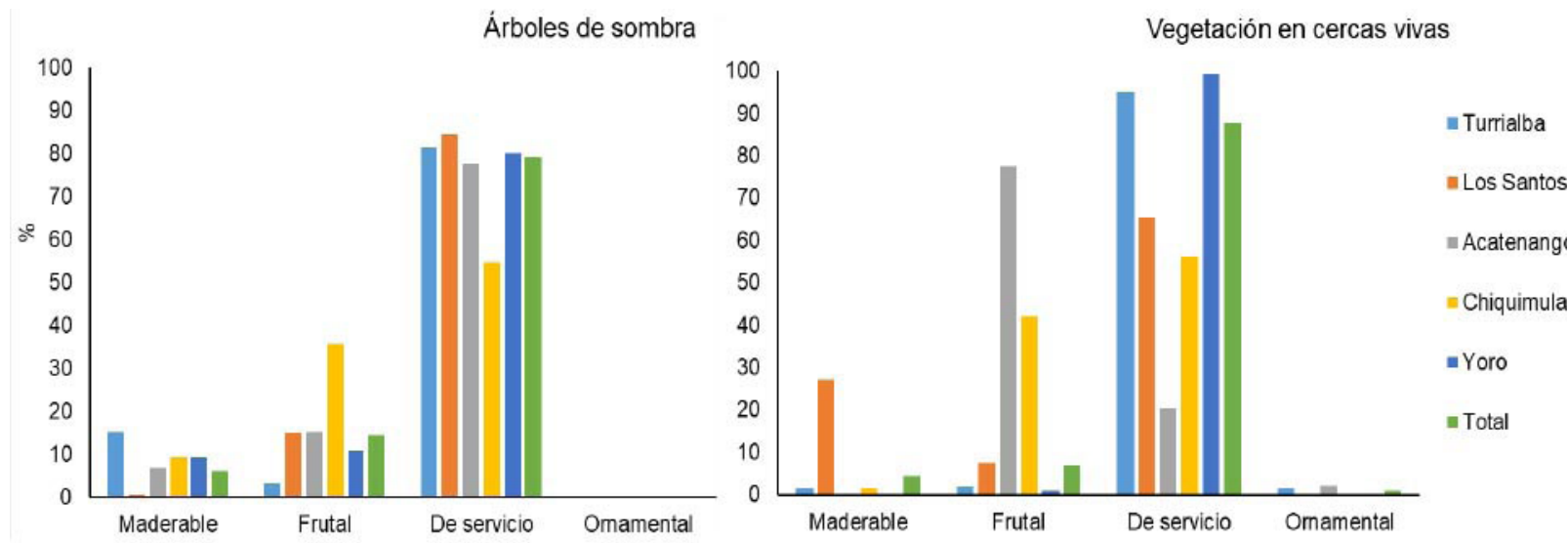

Figura 2. Características de los árboles de sombra registrados $(n=7665)$ en las parcelas de café y la vegetación de cercas vivas $(n=$ 5076) en fincas de pequeños productores de café en cinco paisajes centroamericanos. Turrialba y Los Santos en Costa Rica, Acatenango y Chiquimula en Guatemala, y Yoro en Honduras. Julio 2014 a junio 2015.

Figure 2. Characteristics of shade trees $(n=7665)$ registered in coffee plots and live fence vegetation $(n=5076)$ in smallholder coffee farms in five Central America landscapes. Turrialba and Los Santos in Costa Rica, Acatenango and Chiquimula in Guatemala, and Yoro in Honduras. July 2014 to June 2015. 


\section{b) Cercas vivas}

Con respecto a la caracterización de las cercas vivas, estas presentaron en promedio una densidad de 391,8 árboles $/ \mathrm{km}$ ( $\pm 16,1$; Cuadro 5). Las cercas vivas del paisaje de Yoro tuvieron una mayor densidad de árboles $/ \mathrm{km}$ en comparación con el resto de los paisajes $\left(\mathrm{F}_{4,89}=8,2 ; \mathrm{p}<0,0001\right)$. Por ejemplo, las cercas vivas de Yoro tuvieron tres veces la cantidad de árboles por km que las cercas en el paisaje de Los Santos (Cuadro 5). La alta densidad arbórea observada en las cercas vivas en las fincas de Yoro no se reflejó necesariamente en la riqueza de especies observada en las mismas, ya que estas tuvieron el menor número de especies en comparación al resto de los paisajes $\left(\mathrm{F}_{3,88}=2,8 ; \mathrm{p}=0,047\right)$ (Cuadro 5). Los árboles de las cercas vivas estudiadas tuvieron diámetros promedios menores que los árboles de sombra usados en las parcelas de café, con $11,39 \mathrm{~cm}( \pm 0,45)$ de dap en todos los paisajes. En general, los árboles de las cercas vivas en Los Santos y Chiquimula tuvieron diámetros mayores que en Turrialba y Yoro $\left(\mathrm{F}_{3,88}=20,31, \mathrm{p}<0,0001\right)$.

Cuadro 5. Características de la vegetación de cercas vivas presentes en fincas de pequeños productores de café en cinco paisajes de Centroamérica. Costa Rica, Guatemala y Honduras. Julio 2014 a junio 2015.

Table 5. Characteristics of vegetation of live fences present in smallholder coffee farms in five Central American landscapes. Costa Rica, Guatemala and Honduras. July 2014 to June 2015.

\begin{tabular}{|c|c|c|c|c|c|c|}
\hline & $\begin{array}{l}\text { Turrialba, } \\
\text { Costa Rica }\end{array}$ & $\begin{array}{l}\text { Los Santos, } \\
\text { Costa Rica }\end{array}$ & $\begin{array}{c}\text { Acatenango, } \\
\text { Guatemala }\end{array}$ & $\begin{array}{l}\text { Chiquimula, } \\
\text { Guatemala }\end{array}$ & $\begin{array}{c}\text { Yoro, } \\
\text { Honduras }\end{array}$ & $\begin{array}{r}\text { Total }(5 \\
\text { paisajes })\end{array}$ \\
\hline n (segmentos) & 82 & 39 & 5 & 26 & 40 & 192 \\
\hline $\begin{array}{l}\text { Densidad arbórea promedio / } \\
\mathrm{km})( \pm \mathrm{EE})\end{array}$ & $390,8 \pm 42,4 b$ & $195,1 \pm 58,5 \mathrm{c}$ & - & $321,3 \pm 67,5 \mathrm{bc}$ & $656,6 \pm 60,0 \mathrm{a}$ & $391,8 \pm 16,1$ \\
\hline $\begin{array}{l}\text { Riqueza de especies / } \\
\text { segmento de } 100 \mathrm{~m}( \pm \mathrm{EE})\end{array}$ & $3,8 \pm 0,32$ a & $3,3 \pm 0,45 \mathrm{ab}$ & - & $3,8 \pm 0,52 \mathrm{a}$ & $2,3 \pm 0,46 \mathrm{~b}$ & $3,4 \pm 0,16$ \\
\hline $\begin{array}{l}\text { Diámetro de árboles (dap; } \\
\mathrm{cm})( \pm \mathrm{EE})\end{array}$ & $8,3 \pm 0,74 \mathrm{~b}$ & $17,8 \pm 1,0 \mathrm{a}$ & - & $15,3 \pm 1,2 \mathrm{a}$ & $7,7 \pm 1,1 \mathrm{~b}$ & $11,4 \pm 0,45$ \\
\hline n (número de individuos) & 2555 & 646 & 93 & 413 & 1369 & 5076 \\
\hline $\begin{array}{l}\text { Seis especies más comunes } \\
\text { (\% del total de árboles) }\end{array}$ & $\begin{array}{l}\text { Erythrina sp. } \\
\text { (Grupo de } E . \\
\text { costaricensis } \\
\text { y } \text { E. berte- } \\
\text { roana }(62,8) ; \\
\text { Gliricidia } \\
\text { sepium }(7,5) ; \\
\text { Conostegia xa- } \\
\text { lapensis }(5,9) ; \\
\text { Miconia } \text { sp. } \\
(5,5) ; \text { Tri- } \\
\text { chanthera } \\
\text { gigantea }(2,5) ; \\
\text { Acnistus arbo- } \\
\text { rescens }(2,2)\end{array}$ & $\begin{array}{c}\text { Erythrina sp. } \\
\text { (Grupo de } E . \\
\text { costaricensis y } \\
\text { E. berteroana) } \\
(39,5) ; \text { Acro- } \\
\text { carpus fraxi- } \\
\text { nifolius }(12,5) ; \\
\text { Eucalyptus sp. } \\
(8,1) ; \text { Myrsine } \\
\text { coriacea }(4,3) ; \\
\text { Cupressus lu- } \\
\text { sitánica }(3,9) ; \\
\text { Miconia sp, } \\
(3,7)\end{array}$ & $\begin{array}{c}\text { Yucca gua- } \\
\text { temalensis } \\
\text { (81,7); Critonia } \\
\text { morifolia }(8,6) ; \\
\text { Grevillea ro- } \\
\text { busta }(4,3) ; \\
\text { Citharexylum } \\
\text { donnell-smithii, } \\
(2,2) ; \text { Cordyli- } \\
\text { ne sp. }(1,1) ; \\
\text { Tabaquillo } \\
(1,1)\end{array}$ & $\begin{array}{c}\text { Yucca gua- } \\
\text { temalen- } \\
\text { sis }(42,6) ; \\
\text { Erythrina sp. } \\
\text { (Grupo de } E \text {. } \\
\text { costaricensis y } \\
\mathrm{E}, \text { berteroana) } \\
(26,4) ; \text { Bursera } \\
\text { simaruba }(5,3) ; \\
\text { Spondias pur- } \\
\text { purea }(3,9) ; \\
\text { Llupur }(3,9) ; \\
\text { Gliricidia se- } \\
\text { pium }(2,9)\end{array}$ & $\begin{array}{c}\text { Yucca gua- } \\
\text { temalen- } \\
\text { sis }(94,2) ; \\
\text { Perymenium } \\
\text { grande }(1,0) ; \\
\text { Bursera si- } \\
\text { maruba }(1,0) ; \\
\text { Jatropha } \\
\text { curcas }(0,5) ; \\
\text { Taporono }(0,5) ; \\
\text { Spondias pur- } \\
\text { purea }(0,4)\end{array}$ & $\begin{array}{l}\text { Erythrina sp. } \\
\text { (Grupo de E. } \\
\text { costaricensis y } \\
\text { E. berteroana) } \\
(38,8) ; \text { Yucca } \\
\text { guatemalen- } \\
\text { sis }(31,1) ; \\
\text { Gliricidia } \\
\text { sepium }(4,1) \text {; } \\
\text { Conostegia xa- } \\
\text { lapensis }(3,4) ; \\
\text { Miconia sp. } \\
(3,3) ; \text { Acrocar- } \\
\text { pus fraxinifo- } \\
\text { lius }(1,6)\end{array}$ \\
\hline
\end{tabular}

Se reportan medias ajustadas y errores estándar (EE). Letras asociadas a las medias indican diferencias estadísticas entre paisajes, basado en ANDEVA $(\mathrm{p}<0,05)$ / Adjusted means and standard errors are reported. Letters associated with means indicate statistical differences across landscapes, based on ANOVA $(\mathrm{p}<0.05)$. 
Entre los 5076 árboles medidos en las cercas vivas se reconocieron 97 especies. Las dos especies más comunes en las cercas vivas fueron E. costaricensis y E. beteroana (también conocidas como poró), las cuales fueron identificadas como una sola especie en campo y en conjunto correspondieron al 38,8\% del total de árboles medidos en los 192 segmentos (Cuadro 5). El izote o itabo y las especies Gliricidia sepium (madero negro), Conostegia xalapensis (purra) y Miconia sp. (lengua de vaca), fueron las más frecuentes después del poró, cada una de ellas con más de 150 individuos medidos en cercas vivas. Tanto en las cercas vivas de las fincas de Turrialba como en las de Los Santos, dominaron los árboles de E. poeppigiana. Después del poró, en Los Santos las especies más frecuentes fueron Acrocarpus fraxinifolius (cedro rosa) y una del género Eucalyptus (eucalipto), mientras que, en Turrialba fueron el laurel y la purra. Aunque la dominancia del poró a través de todos los paisajes, como elemento clave de las cercas vivas, estuvo bien reflejada en los paisajes de Costa Rica, la especie más abundante en los paisajes restantes (Acatenango, Chiquimula y Yoro) fue izote o itabo. El poró y Bursera simaruba (indio desnudo, jiote o mulato) fueron las dos especies más abundantes después del izote en Chiquimula. En Acatenango estas dos especies en orden descendente correspondieron a Critonia morifolia y gravilea, con $8,6 \%$ y 4,3\%, respectivamente, del total individuos registrados en las cercas vivas. En Yoro otras especies aparte del izote tuvieron abundancias relativas muy bajas $(\leq 1 \%)$.

La mayoría de los individuos en las cercas vivas fueron reconocidos como especies de servicio (87,8\%; Figura 2). Además, el 7\% correspondieron a árboles frutales y el 4,4\% a árboles maderables. Entre todos los individuos medidos menos del 1\% correspondieron a ornamentales. En Yoro y Turrialba, se observaron las mayores proporciones de árboles de servicio (99,1 y 94,9\%, respectivamente), mientras que, en las fincas de Acatenango fueron menos del 1\% (Figura 2). En las fincas de Acatenango se encontró la mayor proporción de árboles frutales $(77,4 \%)$ y en Turrialba y Yoro la menor (1,9 y 0,9\%, respectivamente). La proporción de árboles maderables fue relativamente baja a través de todos los paisajes, pero se observó una mayor proporción en Los Santos (27,1\%), en comparación con el resto de los paisajes (Figura 2).

\section{c) Otras prácticas de $\mathrm{AbE}$}

Con respecto al resto de las prácticas de $\mathrm{AbE}$, la comparación del área destinada a las mismas, mostró diferencias entre huertos caseros entre paisajes (Cuadro 6). Los productores de Yoro destinaron una mayor área a esta práctica en comparación a los productores de Los Santos y Chiquimula $\left(\mathrm{F}_{3,100}=17,19, \mathrm{p}<0,0001\right)$. No hubo diferencias significativas entre paisajes con respecto al área destinada a barbechos y bosque (Cuadro 6), y debido a

Cuadro 6. Comparación del área promedio ( \pm E.E.) destinada al uso de huertos caseros, barbechos y bosques en fincas de pequeños productores de café en cinco paisajes de Centroamérica. Turrialba y Los Santos en Costa Rica, Acatenango y Chiquimula en Guatemala, y Yoro en Honduras. Julio 2014 a junio 2015.

Table 6. Comparison of mean area $( \pm$ S.E. $)$ dedicated to home gardens, fallows and forests in smallholder coffee farms in five Central American landscapes. Turrialba and Los Santos in Costa Rica, Acatenango and Chiquimula in Guatemala, and Yoro in Honduras. July 2014 to June 2015.

\begin{tabular}{lcccccc}
\hline & $\begin{array}{c}\text { Turrialba, } \\
\text { Costa Rica }\end{array}$ & $\begin{array}{c}\text { Los Santos, } \\
\text { Costa Rica }\end{array}$ & $\begin{array}{c}\text { Acatenango, } \\
\text { Guatemala }\end{array}$ & $\begin{array}{c}\text { Chiquimula, } \\
\text { Guatemala }\end{array}$ & $\begin{array}{c}\text { Yoro, } \\
\text { Honduras }\end{array}$ & $\begin{array}{c}\text { Total } \\
\text { (5 paisajes) }\end{array}$ \\
\hline n (fincas) & $\mathbf{5 0}$ & $\mathbf{5 0}$ & $\mathbf{4 2}$ & $\mathbf{2 0}$ & $\mathbf{2 9}$ & $\mathbf{1 9 1}$ \\
\hline Huertos caseros (ha) & $0,05 \pm 0,02 \mathrm{ab}$ & $0,01 \pm 0,00 \mathrm{~b}$ & - & $0,02 \pm 0,01 \mathrm{~b}$ & $0,10 \pm 0,01 \mathrm{a}$ & $0,05 \pm 003$ \\
Barbecho (ha) & $0,27 \pm 0,08 \mathrm{a}$ & $0,35 \pm 0,11 \mathrm{a}$ & - & - & $0,53 \pm 0,07 \mathrm{a}$ & $0,43 \pm 0,01$ \\
Bosque (ha) & $0,69 \pm 0,22 \mathrm{a}$ & $2,13 \pm 0,44 \mathrm{a}$ & - & - & $2,21 \pm 0,39 \mathrm{a}$ & $1,17 \pm 0,04$ \\
\hline
\end{tabular}

Letras asociadas a las medias indican diferencias estadísticas entre paisajes, basado en ANDEVA $(\mathrm{p}<0,05) /$ Letters associated with means indicate statistical differences across landscapes, based on ANOVA $(\mathrm{p}<0.05)$. 
la baja frecuencia de productores con plantaciones forestales (1,6\% del total de encuestados) y bosques ribereños (9,4\% del total de productores encuestados), estas dos prácticas no se compararon entre paisajes.

\section{Discusión}

Este estudio indica que muchos pequeños productores de café en Costa Rica, Honduras y Guatemala utilizan prácticas de Adaptación basada en Ecosistemas (AbE) en sus fincas. De los 191 productores de café entrevistados, el $100 \%$ hace uso de al menos una práctica de AbE y el $40 \%$ hace uso de cinco o más de estas prácticas, mientras que, el número promedio de prácticas de $\mathrm{AbE}$ promedio por finca fue de 3,68. Algunas prácticas de $\mathrm{AbE}$ (como el uso de árboles dispersos y los surcos en contorno) fueron muy comunes y utilizadas en todos los paisajes, mientras que otras, como el uso de plantaciones forestales, cortinas rompevientos y cobertura viva fueron menos usadas.

Aunque este estudio se enfocó en cinco paisajes cafetaleros centroamericanos, es posible que estos hallazgos sean ampliamente representativos de fincas de pequeños productores de café a lo largo de la región, ya que las características de las fincas y finqueros encuestados son muy similares a aquellas reportadas en otros estudios sobre producción de café a pequeña escala en Nicaragua (Haggar et al., 2015), Costa Rica (Cerdán et al., 2012; Haggar et al., 2015), Guatemala (Haggar et al., 2015) y El Salvador (Méndez et al., 2007). Además, existen muchos otros estudios que resaltan la prevalencia de sistemas de café bajo sombra a lo largo de la región (e.j. Méndez et al., 2007; Haggar et al., 2015), que coinciden con los hallazgos de esta investigación en cuanto a que el uso de árboles de sombra en café es la práctica de AbE más común entre los paisajes estudiados.

Aunque este estudio sugiere que el uso de prácticas de AbE es común entre pequeños productores de café en Centroamérica, existe mucha variación entre fincas y paisajes en las prácticas que están siendo implementadas. El número promedio y el tipo de prácticas de $\mathrm{AbE}$ utilizadas varían entre paisajes. Por ejemplo, fincas en Yoro y Los Santos tuvieron un promedio más alto de prácticas de AbE que los otros paisajes. La variación en el uso y características de estas, refleja las diversas condiciones biofísicas (terreno, clima), factores culturales (preferencia por ciertas especies) y/o la presencia de proyectos y programas de extensión agrícola (tipo de manejo, especies utilizadas, etc.), que en conjunto son factores reconocidos por afectar la toma de decisiones de los finqueros con respecto al manejo de la finca y los cultivos (Harvey et al., 2017).

Estudios en otras regiones han resaltado la alta variación en las prácticas de manejo entre fincas individuales y paisajes, y la importancia de características biofísicas y socioeconómicas que influencian estas prácticas. Por ejemplo, se ha demostrado que factores como el tamaño de la finca (Ithinji, 2011), la edad de los agricultores (Thangata y Alavalapati, 2003; Hisali et al., 2011), el nivel de educación (Knowler y Bradshaw, 2007; Deressa et al., 2011; Below et al., 2012) y la tenencia de la tierra (Erenstein, 2003; Hellin y Schrader, 2003; Hisali et al., 2011; Wall, 2007; Hellin y López-Ridaura, 2016), intervienen en el tipo y uso de práctica de manejo. Los resultados del presente estudio sugieren que aspectos que variaron entre fincas, como el tipo de terreno, el clima y la prevalencia histórica de ciertos tipos de cultivos, podrían estar relacionados con la prevalencia de AbE y el modo específico en que diferentes prácticas de AbE son implementadas. Por ejemplo, los paisajes de Los Santos, Acatenango y Yoro se encuentran en áreas montañosas de topografía más escarpada en comparación al resto de los paisajes. Hay zonas tradicionalmente cafetaleras (ej. Turrialba y Los Santos) y otras en donde el cultivo del café es menos relevante y más reciente (ej. Chiquimula). También existe variación climática entre paisajes, algunos con lluvia estacional marcada (ej. Chiquimula) y otros donde la lluvia es constante a lo largo del año (ej. Turrialba). Todas estas diferencias entre paisajes pueden influir en la selección de prácticas de AbE y usos de las mismas. Sin embargo, aunque se demuestra la prevalencia de las prácticas de AbE en pequeñas fincas de café, una limitante del estudio es que no se contó con información sobre los factores que influyen el uso de estas prácticas. Se requieren estudios puntuales para identificar los factores específicos asociados al uso de AbE o al uso de ciertas prácticas. 
El presente estudio tiene implicaciones clave acerca de los esfuerzos necesarios para promocionar la implementación de AbE. En primer lugar, el hecho de que muchos agricultores ya tengan AbE en sus fincas y tengan experiencia, significa que los profesionales y los agricultores pueden aprender de las experiencias existentes, organizar visitas a las fincas y compartir conocimientos entre los agricultores. En especial, será importante hacer intercambios entre productores que no tienen las prácticas de $\mathrm{AbE}$ con los que las tienen, para que puedan compartir sus experiencias de los efectos de diferentes prácticas sobre la producción y resiliencia del café. Se ha demostrado que estos tipos de experiencias de aprendizaje participativo son muy efectivas para promover la adopción de nuevas prácticas en otros lados (Percy, 1999; Braun et al., 2000; Holt-Giménez, 2002).

En segundo lugar, existe la posibilidad de aumentar el uso de las prácticas de AbE en ciertas regiones y fincas. Por ejemplo, los agricultores de Acatenango implementaron menos prácticas de $\mathrm{AbE} \mathrm{(} 2$ prácticas por finca) que los agricultores en otras regiones (en este paisaje menos del 10\% tienen huerto casero, cercas vivas y terrazas, y no se reporta el uso de parches de bosque, bosque ribereño y plantaciones forestales), lo que sugiere que este paisaje podría requerir atención adicional para promover el uso de AbE. De igual manera, algunas prácticas de EbA como el uso de cobertura viva no son muy comunes en las fincas cafetaleras a pesar de su potencial utilidad (Harvey et al., 2005), y su uso podría promoverse más en fincas y paisajes con ciertas características. El uso de AbE puede ser promovido a través de muchas formas, incluyendo el apoyo técnico, su incorporación en planeamiento territorial, la capacitación, incentivos financieros para la adopción de nuevas prácticas y otros medios, los cuales han demostrado mejorar la adopción en otros lugares (Tucker et al., 2010; Deressa et al., 2011; Bryan et al., 2013; Geneletti y Zardo 2016). En especial, se requiere que los extensionistas y técnicos que atienden a los pequeños productores estén familiarizados con las prácticas de AbE y la habilidad de las mismas para ayudar a los finqueros a adaptarse al cambio climático, así como con otros cobeneficios explícitos de las prácticas (ej. ambientales y socioeconómicos) (Geneletti y Zardo, 2016), y que sean capaces de proveer soporte técnico en la implementación y manejo de estas prácticas.

En tercer lugar, dadas las diferencias en la composición de especies entre paisajes, es importante asegurarse que las especies que promueven los proyectos de adaptación estén adecuadas a las condiciones particulares del paisaje objetivo. Sin embargo, se necesita aún más conocimiento sobre la composición y diversidad de especies utilizadas, y sobre cómo establecer y manejar nuevas prácticas de AbE, para poder lograr que brinden el mayor beneficio posible, tanto a nivel adaptivo como productivo (Harvey et al., 2017).

Finalmente, los resultados de este estudio podrían ser generalizados a otros paisajes de pequeños cafetaleros que dominan gran parte de Centroamérica, ya que los paisajes evaluados fueron seleccionados para ser representativos de la región y cubrir las principales condiciones biofísicas presentes, desde bosques tropicales secos hasta bosques tropicales muy húmedos. Esto es particularmente cierto para algunas prácticas como son el uso de árboles de sombra (Valencia et al., 2014; Haggar et al., 2015) y el uso de cercas vivas (Harvey et al., 2005), las cuales han sido previamente documentadas en la región. Sin embargo, para otras prácticas como son la conservación de fragmentos de bosque y bosques ribereños, y el uso de barbechos, cobertura viva y cortinas rompevientos, existe poca información y es más difícil concluir si los resultados de este estudio son representativos de la región.

\section{Conclusiones}

El estudio indicó que muchos pequeños productores de café en Centroamérica hacen uso de prácticas de AbE, particularmente árboles de sombra y cercas vivas, con un promedio de cuatro prácticas por finca. Sin embargo, hay diferencias significativas entre fincas y paisajes en la prevalencia de prácticas AbE y en cómo cada una de ellas está siendo implementada. Aunque se requiere de más estudios para entender por qué los agricultores están utilizando ciertas prácticas y qué factores influyen en su implementación, este estudio proporciona la primera evaluación de la 
prevalencia de Adaptación basada en Ecosistemas (AbE) en pequeños agricultores, y puede servir como línea base para responsables de políticas y profesionales interesados en promover un mayor uso de AbE.

\section{Agradecimientos}

Los autores agradecen a Jacques Avelino, Bruno Rapidel, Raffaele Vignola y Camila Donatti por sus contribuciones al diseño de la investigación, a Kellee Koening por la ayuda en la preparación de la Figura 1, Nishina Nambiar por su cooperación en la búsqueda de literatura, y a Vlasova González por su apoyo logístico y administrativo.

\section{Literatura citada}

Aguilar, E., T.C. Peterson, P. Ramírez-Obando, R. Frutos, J.A. Retana, M. Solera, J. Soley, I. González-García, R.M. Araujo, A. Rosa-Santos, V.E. Valle, M. Brunet, L. Aguilar, L. Álvarez, M. Bautista, C. Castañón, L. Herrera, E. Ruano, J.J. Sinay, E. Sánchez, G.I. Hernández-Oviedo, F. Obed, J.E. Salgado, J.L. Vázquez, M. Baca, M. Gutiérrez, C. Centella, J. Espinosa, D. Martínez, B. Olmedo, C E. Ojeda-Espinoza, R. Núñez, M. Haylock, H. Benavides, and R. Mayorga. 2005. Changes in precipitation and temperature extremes in Central America and northern South America, 1961-2003. J. Geophys. Res. 110:D23107. doi:10.1029/2005JD006119

Avelino, J., M. Cristancho, S. Georgiou, P. Imbach, L. Aguilar, G. Bornemann, P. Läderach, F. Anzueto, A.J. Hruska, and C. Morales. 2015. The coffee rust crises in Colombia and Central America (2008-2013): impacts, plausible causes and proposed solutions. Food Secur. 7:303-321. doi:10.1007/s12571-015-0446-9

Baca, M., P. Läderach, J. Haggar, G. Schroth, and O. Ovalle. 2014. An integrated framework for assessing vulnerability to climate change and developing adaptation strategies for coffee growing families in Mesoamerica. PLoS One 9:e88463. doi:10.1371/journal.pone.0088463

Bautista-Solís, P., R. Vignola, C.A. Harvey, J. Avelino, M. Chacón, R. Martínez, L. Trevejo, and B. Rapidel. 2014. Contribution of sustainable agricultural management practices to reducing the impacts of extreme weather events in Tropical America. In: C.A. Harvey, and F. Alpizar, editors, CASCADE Project Working Paper. CATIE, Turrialba, CRC. p. 86.

Below, T.B., K.D. Mutabazi, D. Kirschke, C. Franke, S. Sieber, R. Siebert, and K. Tscherning. 2012. Can farmers' adaptation to climate change be explained by socio-economic household-level variables? Glob. Environ. Change 22:223-235. doi:10.1016/j.gloenvcha.2011.11.012

Braun,A.R., G. Thiele, and M. Fernández. 2000. Farmer field schools and local agricultural research committees: complementary platforms for integrated decision-making in sustainable agriculture. Paper $\mathrm{N}^{\circ} 105$. Overseas Development Institute, London, GBR.

Bunn, C., P. Läderach, O. Ovalle-Rivera, and D. Kirschke. 2015. A bitter cup: climate change profile of global production of Arabica and Robusta coffee. Climatic Change 129:89-101. doi:10.1007/s10584-014-1306-x

Bryan, E., C. Ringler, B. Okoba, C. Roncoli, S. Silvestri, and M. Herrero. 2013. Adapting agriculture to climate change in Kenya: household strategies and determinants. J. Environ. Manag. 114:26-35. doi:10.1016/j.jenvman.2012.10.036

Burnham, M., and Z. Ma. 2015. Linking smallholder farmer climate change adaptation decisions to development. Climate Dev. 8:289-311. doi:10.1080/17565529.2015.1067180

Capon, S.J., L.E. Chambers, R. Mac-Nally, R.J. Naiman, P. Davies, N. Marshall, J. Pittock, M. Reid, T. Capon, M. Douglas, J. Catford, D.S. Baldwin, M. Stewardson, J. Roberts, M. Persons, and S.E. Williams. 2013. Riparian ecosystems in the 21st century: hotspots for climate change adaptation? Ecosystems 16:359-381. doi:10-1007/s10021-013-9656-1 
Cerdán, C.R., M.C. Rebolledo, G. Soto, B. Rapidel, and F.L. Sinclair. 2012. Local knowledge of impacts of tree cover on ecosystem services in smallholder coffee production systems. Agric. Syst. 110:119-130. doi:10.1016/j.agsy.2012.03.014

Damon, A. 2000. A review of the biology and control of the coffee berry borer, Hypothenemus hampei (Coleoptera: Scolytidae). Bull. Entomol. Res. 90:453-465. doi:10.1017/S0007485300000584

Dabney, S.M. 1998. Cover crop impacts on watershed hydrology. J. Soil Water Conserv. 53:207-213.

Deressa, T.T., R.M. Hassan, and C. Ringler. 2011. Perception of and adaptation to climate change by farmers in the Nile basin of Ethiopia. J. Agric. Sci. 149:23-31. doi:10.1017/S0021859610000687

Dinesh, D., S. Vermeulen, I. Bacudo, D. Martinez-Baron, A. Castro-Nunez, M. Hedger, S. Huyer, P. Iversen, A. Laure, A.M. Loboguerrero, C. Martius, H Neufeldt, M. Nyasimi, M. Richards, and L. Wollenberg. 2016. Options for agriculture at marrakech climate talks: messages for SBSTA 45 agriculture negotiators. CCAFS Report No. 6. Climate Change, Agriculture and Food Security (CCAFS), Copenhagen, DEN.

Di-Rienzo, J.A., F. Casanoves, M.G. Balzarini, L. González, M. Tablada, y C.W. Robledo. 2016. InfoStat. Universidad Nacional de Córdoba, ARG.

Donatti, C.I., C.A. Harvey, M.R. Martínez-Rodríguez, R. Vignola, and C.M. Rodríguez. 2017. What information do policy makers need to develop climate adaptation plans for smallholder farmers? The case of Central America and Mexico. Clim. Change 141:107-121. doi:10.1007/s10584-016-1787-x

Easterling, W.E., C.J. Hays, M. McKenney-Easterling, and J.R. Brandle. 1997. Modelling the effect of shelterbelts on maize productivity under climate change: an application of the EPIC model. Agric. Ecosyst. Environ. 61:163-176. doi:10.1016/ S0167-8809(96)01098-5

Erenstein, O. 2003. Smallholder conservation farming in the tropics and sub-tropics: a guide to the development and dissemination of mulching with crop residues and cover crops. Agric. Ecosyst. Environ. 100:17-37. doi:10.1016/S0167-8809(03)00150-6

Gay, C., F. Estrada, C. Conde, H. Eakin, and L. Villers. 2006. Potential impacts of climate change on agriculture: a case study of coffee production in Veracruz, Mexico. Climatic Change 79:259-288. doi:10.1007/s10584-006-9066-x

Geneletti, D., and L. Zardo. 2016. Ecosystem-based adaptation in cities: An analysis of European urban climate adaptation plans. Land Use Policy 50:38-47. doi:10.1016/j.landusepol.2015.09.003

Graeub, B.E., M. Jahi-Chappell, H. Wittman, S. Ledermann, R. Bezner-Kerr, and B. Gemmill-Herren. 2016. The state of family farms in the world. World Dev. 87:1-15. doi:10.1016/j.worlddev.2015.05.012

Giorgi, F. 2006. Climate change hot-spots. Geophys. Res. Lett. 33:L08707. doi:10.1029/2006GL025734

Haggar, J., M. Asigbaase, G. Bonilla, J. Pico, and A. Quilo. 2015. Tree diversity on sustainably certified and conventional coffee farms in Central America. Biodivers. Conserv. 24:1175-1194. doi:10.1007/s10531-014-0851-y

Hannah, L., C.I. Donatti, C.A. Harvey, E. Alfaro, D.A. Rodriguez, C. Bouroncle, E. Castellanos, F. Dias, E. Fung, H.G. Hidalgo, P. Imbach, P. Läderach, J. Landrum, and A.L. Solano. 2017. Regional modeling of climate change influence on ecosystems and smallholder agriculture in Central America. Climatic Change 141:63-75. doi:10.1007/s10584-016-1867

Harvey, C.A., M.R. Martínez-Rodríguez, J.M. Cárdenas, J. Avelino, B. Rapidel, R. Vignola, C.I. Donatti, and S. Vilchez Mendoza. 2017. The use of Ecosystem-based Adaptation practices by smallholder farmers in Central America. Agric. Ecosyst. Environ. 246:279-290. doi:10.1016/j.agee.2017.04.018

Harvey, C.A., M. Saborio-Rodríguez, M.R. Martinez-Rodríguez, B. Viguera, A. Chain, R. Vignola, and F. Alpizar. 2018. Climate change impacts and adaptation among smallholder farmers in Central America. Agric. Food Secur. 7:57. doi:10.1186/s40066-018-0209-x 
Harvey, C.A., C. Villanueva, J. Villacís, M. Chacón, D. Muñoz, M. López, M. Ibrahim, R. Gómez, R. Taylor, J. Martinez, A. Navas, J. Sáenz, D. Sánchez, A. Medina, S. Vilchez, B. Hernández, A. Perez, F. Ruiz, F. López, I. Lang, and F.L. Sinclair. 2005. Contribution of live fences to the ecological integrity of agricultural landscapes. Agric. Ecosyst. Environ. 111:200230. doi:10.1016/j.agee.2005.06.011

Hellin, J., and S. López-Ridaura. 2016. Soil and water conservation on Central American hillsides: if more technologies is the answer, what is the question? AIMS Agric. Food 1:194-207. doi:10.3934/agrfood.2016.2.194

Hellin, J., and K. Schrader. 2003. The case against direct incentives and the search for alternative approaches to better land management in Central America. Agric. Ecosyst. Environ. 99:61-81. doi:10.1016/S0167-8809(03)00149-X

Hisali, E., P. Birungi, and F. Buyinza. 2011. Adaptation to climate change in Uganda: evidence from micro level data. Glob. Environ. Change 21:1245-1261. doi:10.1016/j.gloenvcha.2011.07.005

Holland, M.B., S.Z. Shamer, P. Imbach, J.C. Zamora, C. Medellin, E.J. Leguia, C.I. Donatti, M.R. Martínez-Rodríguez, and C.A. Harvey. 2017. Mapping agriculture and adaptive capacity: applying expert knowledge at the landscape scale. Climatic Change 141:139-153. doi:10.1007/s10584-016-1810-2

Holt-Giménez, E. 2002. Measuring farmers' agroecological resistance after Hurricane Mitch in Nicaragua: a case study in participatory, sustainable land management impact monitoring. Agric. Ecosyst. Environ. 93:87-105. doi:10.1016/S01678809(02)00006-3

Imbach, P., B. Locatelli, J.C. Zamora, E. Fung, L. Calderer, L. Molina, and P. Ciais. 2015. Impacts of climate change on ecosystem hydrological services of Central America: water availability. In: A. Chiabai, editor, Climate change impacts on tropical forests in Central America: an ecosystem service perspective. Earthscan, NY, USA. p. 65-90.

Imbach, P., L. Molina, B. Locatelli, O. Roupsard, G. Mahé, R. Neilson, L. Corrales, M. Scholze, and P. Ciais. 2012. Modeling potential equilibrium states of vegetation and terrestrial water cycle of Mesoamerica under climate change scenarios. J. Hydrometeorol. 13:665-680. doi:10.1175/JHM-D-11-023.1

Ithinji, G.K. 2011. Determinants of adaptation of shade coffee technology and the role of agroforestry in the productivity and profitability of coffee in South District, Kenya. PhD. Diss., Egerton University, Nairobi, KEN.

Jha, S., C.M. Bacon, S.M. Philpott, R.A. Rice, V.E. Méndez, and P. Läderach. 2011. A review of ecosystem services, farmer livelihoods, and value chains in shade coffee agroecosystems. In: W.B. Campbell, and S. López-Ortíz, editors, Agroecology: Integrating agriculture, conservation and ecotourism: examples from the field-present status and future prospectus. Springer, Dordrecht, HOL. p. 141-208.

Jones, P.G., and P.K. Thornton. 2003. The potential impacts of climate change on maize production in Africa and Latin America in 2055. Glob. Environ. Change 13:51-59. doi:10.1016/S0959-3780(02)00090-0

Knowler, D., and B. Bradshaw. 2007. Farmers' adoption of conservation agriculture: a review and synthesis of recent research. Food Policy 21:25-48. doi:10.1016/j.foodpol.2006.01.003

Lin, B.B. 2011. Resilience in agriculture through crop diversification: adaptive management for environmental change. BioScience 61:183-193. doi:10.1525/bio.2011.61.3.4

Lin, B.B. 2007. Agroforestry management as an adaptive strategy against potential microclimate extremes in coffee agriculture. Agric. For. Meteorol. 144: 85-94. doi:10.1016/j.agrformet.2006.12.009

Locatelli, B., V. Evans, D.A. Wardell, A. Andrade, and R. Vignola. 2011. Forests and climate change in Latin America: linking adaptation and mitigation. Forests 2:431-450. doi:10.3390/f2010431

Magrach, A., and J. Ghazoul. 2015. Climate and pest-driven geographic shifts in global coffee production: Implications for forest cover, biodiversity and carbon storage. PLoS One 10(7):e0133071. doi:10.1371/journal.pone.0133071

Méndez, V.E., S.R. Gliessman, and G.S. Gilbert. 2007. Tree biodiversity in farmer cooperatives of a shade coffee landscape in western El Salvador. Agric. Ecosyst. Environ. 119:145-159. doi:10.1016/j.agee.2006.07.004 
Morton, J.F. 2007. The impact of climate change on smallholder and subsistence agriculture. PNAS 104:19680-19685. doi:10.1073/pnas.0701855104

Neelin, J.D., M. Münnich, H. Su, J.E. Meyerson, and C.E. Holloway. 2006. Tropical drying trends in global warming models and observations. PNAS 103:6110-6115. doi:10.1073/pnas.0601798103

Ovalle-Rivera, O., P. Läderach, C. Bunn, M. Oberstainer, and G. Schroth. 2015. Projected shifts in Coffea arabica suitability among major global producing regions due to climate change. PLoSONE 10(4):e0124155. doi:10.1371/journal.pone.0124155

Percy, R. 1999. The experiential learning cycle and its application towards the transformation of governmental extension services in sub-Saharan Africa. Int. J. Lifelong Edu. 18:370-384. doi:10.1080/026013799293612

Peters, V.E., and C.R. Carroll. 2012. Temporal variation in coffee flowering may influence the effects of bee species richness and abundance on coffee production. Agroforest. Syst. 85:95-103. doi:10.1007/s10457-011-9476-2.

PRESANCA II (Programa Regional de Seguridad Alimentaria de Centroamérica), y FAO. 2011. Centroamérica en cifras: datos de seguridad alimentaria nutricional y agricultura familiar. FAO, Roma, ITA. http://www.fao.org/fileadmin/user_upload/ AGRO_Noticias/docs/CentroAm\%C3\%A9ricaEnCifras.pdf. (consultado 10 oct. 2017).

Rosenberg, N. 1992. Adaptation of agriculture to climate change. Climatic Change 21:385-405. doi:10.1007/BF00141378

Schroth, G., P. Laderach, J. Dempewolf, S. Philpott, J. Haggar, H. Eakin, T. Castillejos, J. Garcia-Moreno, L. Soto-Pinto, R. Hernandez, A. Eitzinger, and J. Ramirez-Villegas. 2009. Towards a climate change adaptation strategy for coffee communities and ecosystems in the Sierra Madre de Chiapas, Mexico. Mitig. Adap. Strategies Glob. Chang. 14:605-625. doi:10.1007/s11027-009-9186-5

Siles, P., J.M. Harmand, and P. Vaast. 2010. Effects of Inga densiflora on the microclimate of coffee (Coffea arabica L.) and overall biomass under optimal growing conditions in Costa Rica. Agroforest. Syst. 78:269-286. doi:10.1007/s10457-009-9241-y

Thangata, P.H., and J.R.R. Alavalapati. 2003. Agroforestry adoption in southern Malawi: the case of mixed intercropping of Gliricidia sepium and maize. Agric. Syst. 78:57-71. doi:10.1016/S0308-521X(03)00032-5

Tucker, C.M., H. Eakin, and E.J. Castellanos. 2010. Perceptions of risk and adaptation: coffee producers market shocks, and extreme weather in Central America and Mexico. Glob. Environ. Change 20:23-32. doi:10.1016/j.gloenvcha.2009.07.006

Valencia, V., L. García-Barrios, P. West, E.J. Sterling, and S. Naeem. 2014. The role of coffee agroforestry in the conservation of tree diversity and community composition of native forests in a Biosphere Reserve. Agric. Ecosyst. Environ. 189:154163. doi:10.1016/j.agee.2014.03.024

Verchot, L.V., M. VanNoordwijk, S. Kandji, T. Tomich, C. Ong, A. Albrecht, J. Mackensen, C. Bantilan, K.V. Anupama, and C. Palm. 2007. Climate change: linking adaptation and mitigation through agroforestry. Mitig. Adapt. Strat. Glob. Change 12:901-918. doi:10.1007/s11027-007-9105-6

Vermeulen, S.J. 2014. Climate change, food security and small-scale producers: Analysis of findings of the fifth assessment report of the Intergovernmental Panel on Climate Change (IPCC). Climate Change, Agriculture and Food Security (CCAFS), Copenhagen, DEN.

Vignola, R., C.A. Harvey, P. Bautista-Solis, J. Avelino, B Rapidel, C. Donatti, and M.R. Martinez. 2015. Ecosystem-based adaptation for smallholder farmers: Definitions, opportunities and constraints. Agric. Ecosyst. Environ. 211:126-132. doi:10.1016/j.agee.2015.05.013

Villers, L., N. Arizpe, R. Orellana, C. Conde, y J. Hernández. 2009. Impactos del cambio climático en la floración y desarrollo del fruto del café en Veracruz, México. Interciencia 34:322-329.

Wall, P.C. 2007. Tailoring conservation agriculture to the needs of small farmers in developing countries: an analysis of issues. J. Crop Improv. 19:137-155. doi:10.1300/j411v19n01_07 